The Egyptian Journal of Hospital Medicine (January 2019) Vol. 74 (6), Page 1337-1347

\title{
Measurement of Epicardial Adipose Tissue Thickness and Vitamin D Status in Obese Egyptian Adolescents and their Relationship with Cardiovascular Dysfunction
}

\author{
Abd Allah Hussein El-Sadek ${ }^{1}$, Mohammed Noshy El-Alfy ${ }^{1}$, Mansour Mohammed Mustafa ${ }^{2}$, Abd Allah \\ Mohammed El-Khshen ${ }^{3}$, Kamel Soliman Hammad ${ }^{4}$, Ahmed Ali Hassan Ali ${ }^{* 1}$ \\ Departments of ${ }^{1}$ Internal Medicine, ${ }^{2}$ Cardiology, ${ }^{3}$ Diagnostic Radiology, ${ }^{4}$ Clinical Pathology, \\ Faculty of Medicine, Al-Azhar University, Cairo, Egypt
}
*Corresponding author: Ahmed Ali Hassan Ali, E-mail: ahmedalyhassan@gmail.com, phone: $+201065235216$

\begin{abstract}
Background: Epicardial Adipose Tissue (EAT) is the cardiac visceral adipose tissue, influencing its structure and function, and easily assessed by trans-thoracic echocardiography. Vitamin D deficiency is prevalent among obese adolescents and is thought to affect the cardiovascular system.

Objective: This study was aimed to assess the EAT thickness (EATT) and Vitamin D status in obese adolescents to clarify their relationship with cardiovascular dysfunctions.

Patients and Methods: This was a case-control study done at the Internal Medicine Outpatient Clinics of Al-Azhar University Hospitals during the period from February 2016 to February 2018. It included 90 ado lescents divided into two equal groups; obese group (45 obese) and healthy non-obese group (45 ones). Hi story taking, clinical examination, laboratory investigations, and echocardiographic assessment were done to retrieve all relevant data.

Results: Obese adolescents were substantially different from non-obese regarding their anthropometrics w ith worse glucose homeostasis, insulin resistance and lipid profile, with a high prevalence of Metabolic Sy ndrome (MetS) (57.8\%). Echocardiographically, they had a significantly increased carotid intima-media th ickness (cIMT) and EATT, higher incidence of hypertrophic cardiac remodeling, impaired systolic and dia stolic LV and RV functions. 25-Hydroxy Vitamin D (25[OH]VD) levels was lower in obese group vs. non -obese (10.2 \pm 3.8 vs. $19.09 \pm 7.8)$ respectively. EATT, cIMT, and s. Adiponectin showed the highest diag nostic capability in identification of Adolescence obesity and MetS.

Conclusion: EATT is an easily obtainable marker of cardiovascular structural and functional derangements. Vitamin D deficiency significantly worsen the cardio-metabolic risk profile and the cardiovascular function among Egyptians obese adolescents.
\end{abstract}

Keywords: Epicardial Adipose Tissue, Vitamin D, Adolescence Obesity, Cardiovascular Dysfunction.

\section{Introduction}

Throughout the past decades, the incidence of adolescent obesity has significantly increased in both developed and developing countries ${ }^{(1,2)}$. Obesity in adolescence usually associated with considerable morbidities that hinder the quality of life ${ }^{(3)}$. These complications not only restricted to adolescence period but also extended to the adulthood which can lead to premature death ${ }^{(\mathbf{4})}$. Additionally, these sequels enhance the incidence of obesity in the next generations ${ }^{(5)}$. The most terrible complications associated with adolescent obesity are elevated blood pressure (BP), diabetes, dyslipidemia that constitutes in the emerging of metabolic syndrome (MetS) ${ }^{(6)}$. Hereafter, it is a strong risk factor in the developing of cardiovascular dysfunction ${ }^{(7)}$. Of note, carotid intima-media thickness (cIMT) is a strongest predictive parameter in the detection of heart attack and stroke in adulthood and is established to be increased among adolescent obese patients ${ }^{(\boldsymbol{8})}$.

The epicardial adipose tissue thickness (EATT) reflects the cardiac and visceral adiposity, and it has been suggested as a new cardiometabolic risk factor owed by its close association with the myocardium status ${ }^{(9)}$. EATT contributed 


\section{El-Sadek et al.}

significantly in increasing the cardiovascular risk due to the capability of adipocytes in the production of interleukin-6, free fatty acids, tumor necrosis factor- $\alpha$ (TNF- $\alpha)$, and plasminogen activator inhibitor ${ }^{(\mathbf{1 0})}$.

The incidence of obesity is established to be significantly associated with vitamin D deficiency (VDD) apart from vitamin D (VD) is involved in cell proliferation, differentiation, apoptosis, and angiogenesis offering a considerable explanation of the pathogenesis of various morbidities such as cardiac dysfunction, hypertension, obesity, and $\mathrm{DM}^{(\mathbf{1 1 )}}$.

The relationship of EATT to cardiac dysfunction and cardiovascular risk factors is still not well understood, especially among obese adolescents and further research is needed before it can be used as a tool for routine clinical assessment. Moreover, the association between EATT and certain surrogate markers of atherosclerosis, such as cIMT, remains unclear. In addition, there is insufficient data on the relationship between EATT and $25[\mathrm{OH}] \mathrm{VD}$ with obesity alone or in association with other features of MetS specially in the adolescent people particularly it is well known that obesity in this age group is not uncommon, overgrowing, and predispose them to cardiovascular risk that is likely to persist during adulthood.

Therefore, this study was carried out to appreciate the association between EATT, VD status and the risk of cardiovascular dysfunction among Egyptian obese adolescents.

\section{Patients and Methods}

This case-control study included a total of 90 adolescents (12-18 years) who were attending at the Internal Medicine Outpatient Clinics of AlAzhar University Hospitals, for evaluation of obesity as well as other recruited volunteers. This study was conducted between February 2016 to February 2018. The study was approved by the Ethics Board of Al-Azhar University.

This study was implemented in conformity with the declaration of Helsinki, and accordance with the ethics approved by the Ethics Unit, Faculty of Medicine, Al-Azhar University. Informed consents were obtained from the included participants and from their parents after explaining the nature of the study to all of them.

Patients were divided into two equal groups according to age and sex-specific (body mass index) BMI percentiles; obese group (45 participants), and non-obese group (45 participants).

\section{Inclusion criteria}

All apparently healthy adolescents aged 12- 18 years; presented with obesity that was documented clinically when they attained a BMI $\geq 95^{\text {th }}$ of age and sex matched percentiles and agreed to participate in the study fulfilled the inclusion criteria. Consequently, non-obese healthy adolescents were eligible for inclusion in the control group. Most of the healthy control subjects were recruited from friends, brothers \& sisters of subjects included in group I as well as some of those who came for routine health care checks, proven free of illnesses and agreed to participate in this study.

\section{Exclusion criteria}

Patients with history of chronic conditions such as bronchial asthma, diabetes Mellitus (Type I or II), hypertension (HTN), psychiatric conditions, endocrinopathies causing obesity like Cushing's syndrome, and hypothyroidism were omitted from the study. Similarly, patients with syndromic obesity, cardiac dysfunction history, chronic kidney disease, chronic liver disease, acute inflammatory diseases, collagen diseases or malignant neoplasms were excluded. Additionally, patients who were receiving medications that are known to affect the tested parameters like; steroids, Vitamin D and/or calcium supplementation, weight reducing agents, anti-lipid agents, and psychotropic medications, and patients with metabolic bone disorders (including parathyroid related problems) were excluded.

\section{Clinical and laboratory evaluation}

To ensure the high levels of quality, all participants were subjected to meticulous history taking, and clinical examination to retrieve all relevant data. Hsitory taking was done to reveal the following data; age, sex, history of cardiac dysfunction, kidney disease, liver dysfunction, 
diabets, endocrne diseases, cancer, and collagen diseases. Consequently, to assess the state of obesity, we evaluated the following parametrs; body weight, height, BMI, waist circumference (WC), systolic blood pressure (SBP), diastolic blood pressure (DBP) according to the age and sex- specific percentiles for each parameter.The stage of pubertal development was determined based on the Tanner staging system ${ }^{(\mathbf{1 2})}$. For each participant, a $10 \mathrm{ml}$ fasting venous blood sample was withdrawn on the same day that the anthropometric data was gathered. The following variables were assessed; Fasting plasma glaucous (FPG), 2 hours post prandial plasma glucose (PPPG), glycated hemoglobin ( $\mathrm{HbA} 1 \mathrm{c} \%)$, fasting insulin, homeostatic model assessment of insulin resistance (HOMA-IR), triglycerides (TG), Total cholesterol (TC), High Density Lipoprotein (HDL-c), Low Density Lipoprotein (LDL-c), $25[\mathrm{OH}] \mathrm{VD}$, adiponectin, high sensitivity CReactive Protein (hs CRP), total calcium, phosphate, and alkaline phosphatase. Furthermore, liver function tests including alanine transaminase (ALT), aspartate transaminase (AST), albumin and creatinine were evaluated.

\section{Radiological assessment Ultrasound examination of carotid intima- media thickness (cIMT)}

For measurement of intimal thickness, we assessed both carotid arteries and detection of atheromatous plaques as a marker of subclinical atherosclerosis. Radiologists scanned the right and left common carotid arteries longitudinally, and the cIMT measurement was taken in the proximal part of the common carotid artery, $1 \mathrm{~cm}$ proximal to the carotid bulb as the maximum distance between the intima-lumen and adventitia-media interfaces. The image was focused on the far wall (dorsal arterial wall) and image quality was optimized with gain settings. All images were taken at end diastole, incident with the R-wave. cIMT was determined as the average of 6 measurements, 3 from each of the left and right common carotid arteries.

\section{Echocardiographic examination}

Conventional transthoracic 2-D echocardiography was performed with ultrasonography (Acuson Sequoia 512 mainframe, Acuson, Mountain View, CA), using a $3.5 \mathrm{MHz}$ scanning frequency phased-array transducer after 15 minutes of resting. Epicardial adipose tissue (EAT) is generally identified as the relatively echo-free space between the outer wall of the myocardium and the visceral layer of pericardium; its thickness was measured during end systole at the point on the free wall of the right ventricle $(\mathrm{RV})$ along the midline of the ultrasound beam, with the best effort to be perpendicular to the aortic annulus, used as an anatomic landmark ${ }^{(13)}$. Standard M-mode recordings of left ventricle (LV) measurements, including LV dimension at end diastole (LVEDD), LV posterior wall thickness at end diastole (LVPWd) and interventricular septal thickness at end diastole (IVSd) were obtained according to the American Society of Echocardiography (ASE) ${ }^{(\mathbf{1 4})}$.

The Ejection fraction (EF) was calculated using the Teichholz method to evaluate the systolic function of the LV ${ }^{(\mathbf{1 5})}$. LVM was calculated from the using Devereux's formula. The Left Ventricular Mass Index (LVMI) $\left(\mathrm{g} / \mathrm{m}^{2.7}\right)$ was corrected for body height in meters to the allometric power of 2.7. Systolic and Diastolic functions of the LV \& RV were assessed by evaluating the corresponding transvalvular (mitral or tricuspid valves) flow as well as their annular tissue velocities parameters obtained from the Pulsed Wave Doppler (PWD) \& the Pulsed Wave-Tissue Doppler Imaging (PW-TDI) methods respectively, through the standard fourchamber and two-chamber views.

\section{Statistical analysis}

Statistical analysis was performed using SPSS software version 23 for Windows (SPSS Inc., Chicago, IL, USA). Normally- distributed data were exemplified in the form of Mean \pm SD and the particular groups were compared using Student's t-test. Moreover, categorical variables were elucidated as number and percentage, and compared using Fisher Exact test or Chi-square test. Correlation analysis was performed using the Pearson's correlation coefficient for continuous normally distributed variables, while the Spearmann's rank correlation was used for other variables. Z-test was used to compare the statistical significance of the difference between two independent correlation coefficients to determine the strength of correlation in either groups. 
Multiple regression analysis was done for detection of the independent predictors of EATT in Adolescents with MetS using $\mathrm{X}^{2}$ and t-test analysis. Receiver operating characteristic (ROC) curve and area under ROC curve (AUC) were used to assess the diagnostic ability of biomarkers using MedCalc software version 14.8 (MedCalc Software, Mariakerke, Belgium). The optimal cut off points were calculated to maximize sensitivity and specificity. All tests were considered significant when $\mathrm{P}<0.05$.

\section{Results}

This study included an overall 90 candidates that were assorted into two equal groups; obese group (45 candidates) and healthy non-obese group (45 candidates) with mean age of $15.5 \pm 1.6$ and $15.16 \pm 1.9$ years in the obese, and non-obese groups, respectively. Subsequently, both groups showed substantially significant difference regarding BMI, body weight, $\mathrm{WC}, \mathrm{SBP}$ and DBP ( $\mathrm{p}<0.0001$ for each parameter). Similarly, Comparison between the two groups regarding the laboratory variables showed a statistically significant difference in glucose homeostasis parameters (FPG \& HbA1c \%), insulin resistance parameters (Fasting insulin \& HOMA-IR), the lipid parameters (TC, LDL-c, HDL-c \& TG), 25[OH]VD, iPTH, and serum adiponectin levels ( $p<0.0001$ for each parameter). Of the included subjects; 26 subjects $(57.8 \%)$ out of the 45 ones included in obese group met the criteria of diagnosis of adolescence MetS while, neither the remaining 19 ones $(42.2 \%)$ within group I of obese adolescents nor any of the subjects of group II did. (Table 1).

\begin{tabular}{|c|c|c|c|}
\hline \multirow{2}{*}{ Variable } & Obese group $(n=45)$ & Non-obese group $(n=45)$ & \multirow{2}{*}{ P-value } \\
\hline & Mean/SD (n. /\%) & Mean/SD (n. / \%) & \\
\hline Ages (years) & $15.6 \pm 1.6$ & $15.16 \pm 1.9$ & 0.281 \\
\hline Weight (Kg) & $84.42 \pm 12.09$ & $56.50 \pm 5.40$ & $<0.0001$ \\
\hline Height (M) & $1.60 \pm 0.06$ & $1.60 \pm 0.05$ & 0.28 \\
\hline BMI $\left(\mathrm{Kg} / \mathrm{m}^{2}\right)$ & $31.50 \pm 3.30$ & $21.50 \pm 1.50$ & $<0.0001$ \\
\hline $\mathrm{WC}(\mathrm{cm})$ & $96.7 \pm 8.50$ & $72.80 \pm 4.40$ & $<0.0001$ \\
\hline SBP (mmHg) & $119.20 \pm 6.20$ & $110.40 \pm 5.90$ & $<0.0001$ \\
\hline DBP (mmHg) & $74.90 \pm 3.20$ & $67.40 \pm 5.70$ & $<0.0001$ \\
\hline MetS & $26(57.8 \%)$ & $0(0 \%)$ & $<0.0001$ \\
\hline FPG (mg/dl) & $91.4 \pm 11.6$ & $77.04 \pm 9.9$ & $<0.0001$ \\
\hline PPBG (mg/dl) & $131.9 \pm 6.8$ & $130.2 \pm 5.1$ & 0.17 \\
\hline HbA1c \% & $5.43 \pm 0.24$ & $5.16 \pm 0.12$ & $<0.0001$ \\
\hline HOMA-IR & $5.3 \pm 1.3$ & $1.7 \pm 0.06$ & $<0.0001$ \\
\hline TG (mg/dl) & $149.2 \pm 18.4$ & $138.2 \pm 9.2$ & $<0.001$ \\
\hline TC (mg/dl) & $203.7 \pm 9.2$ & $184.4 \pm 4.9$ & $<0.0001$ \\
\hline LDL-c (mg/dl) & $131.3 \pm 11.7$ & $99.5 \pm 6.05$ & $<0.0001$ \\
\hline HDL-c (mg/dl) & $42.5 \pm 6.2$ & $57.2 \pm 5.8$ & $<0.0001$ \\
\hline $25[\mathrm{oh}] \mathrm{VD}(\mathrm{ng} / \mathrm{mL})$ & $10.2 \pm 3.8$ & $19.09 \pm 7.8$ & $<0.0001$ \\
\hline iPTH (pg/mL) & $57.3 \pm 15.09$ & $48.18 \pm 20.8$ & 0.021 \\
\hline Hs-CRP (mg/dl) & $2.5 \pm 0.05$ & $0.67 \pm 0.1$ & $<0.0001$ \\
\hline Adiponectin $(\mu \mathrm{g} / \mathrm{mL})$ & $9.5 \pm 2.8$ & $17.8 \pm 2.08$ & $<0.0001$ \\
\hline
\end{tabular}

Abbreviations; $\mathbf{B M I}=$ body mass index, $\mathbf{W C}=$ waist circumference, $\mathbf{S B P}=$ systolic blood pressure, $\mathbf{D B P}=$ diastolic blood pressure, $\mathbf{F P G}=$ fasting plasma glaucous, $\mathbf{2 H P P B G}=$ two hours post-prandial blood glucose, HbA1C= glycosylated hemoglobin, HOMA-IR= homeostatic model assessment of insulin resistance, $\mathbf{T G}=$ triglyceride, $\mathbf{T C}=$ total cholesterol, $\mathbf{L D L}=$ low density lipoprotein, $\mathbf{H D L}=$ high density lipoprotein , iPTH= intact parathormone hormone, HS-CRP=high sensitivity C-reactive protein. 
There was a statistically significant difference between the two groups regarding cIMT , EATT, measurements of the structural dimensions of the LV including (LVEDD, LVDPWT, IVSDT), the LVM \& LVMI, the systolic and diastolic function parameters obtained from the Pulsed Wave Doppler (PWD) \& the Pulsed WaveTissue Doppler Imaging (PW-TDI) methods respectively ( $\mathrm{p}<0.0001$ for each parameter).

(Table 2).

\begin{tabular}{|c|c|c|c|}
\hline \multirow{2}{*}{ Variable } & Obese group $(n=45)$ & Non-obese group $(n=45)$ & \multirow{2}{*}{ P-value } \\
\hline & Mean/SD (n. / \%) & Mean/SD (n. / \%) & \\
\hline cIMT (mm) & $1.17 \pm 0.27$ & $0.56 \pm 0.15$ & $<0.0001$ \\
\hline EATT (mm) & $8.88 \pm 1.1$ & $4.69 \pm 1.41$ & $<0.0001$ \\
\hline LVEDD (mm) & $44.70 \pm 1.77$ & $39.17 \pm 1.13$ & 0.004 \\
\hline LVDPWT (mm) & $10.73 \pm 0.77$ & $8.60 \pm 0.76$ & $<0.0001$ \\
\hline IVSDT (mm) & $10.48 \pm 0.33$ & $8.85 \pm 0.81$ & $<0.001$ \\
\hline $\operatorname{LVMI}\left(\mathrm{g} / \mathrm{m}^{2.7}\right)$ & $43.03 \pm 7.3$ & $38.1 \pm 5.3$ & 0.004 \\
\hline$(\mathrm{M}) \mathbf{E}(\mathrm{cm} / \mathrm{s})$ & $106.82 \pm 4.82$ & $118.35 \pm 4.73$ & $<0.0001$ \\
\hline (M) A (cm/s) & $61.94 \pm 3.40$ & $53.20 \pm 2.36$ & $<0.0001$ \\
\hline (M) E/A & $1.7 \pm 0.17$ & $2.2 \pm 0.1$ & $<0.0001$ \\
\hline DT (m.sec) & $224.04 \pm 3.41$ & $208.49 \pm 7.80$ & $<0.0001$ \\
\hline (M) $\mathrm{E}^{\prime}(\mathrm{cm} / \mathrm{s})$ & $15.31 \pm 1.26$ & $18.14 \pm 0.95$ & $<0.0001$ \\
\hline$(\mathrm{M}) \mathrm{A}^{\prime}(\mathrm{cm} / \mathrm{s})$ & $7.5 \pm 0.5$ & $6.4 \pm 0.6$ & 0.001 \\
\hline (M) $\mathrm{E}^{\prime} / \mathrm{A}^{\prime}$ & $2.06 \pm 0.32$ & $2.84 \pm 0.38$ & $<0.0001$ \\
\hline (M) S (cm/s) & $6.1 \pm 0.55$ & $9.27 \pm 1.23$ & $<0.001$ \\
\hline$(\mathbf{L V}) \mathrm{E} / \mathrm{E}^{\prime}$ & $7 \pm 0.29$ & $6.49 \pm 0.13$ & $<0.001$ \\
\hline$(\mathrm{T}) \mathrm{E}^{\prime}(\mathrm{cm} / \mathrm{s})$ & $13.14 \pm 1.18$ & $15.88 \pm 0.67$ & $<0.001$ \\
\hline (T) $A^{\prime}(\mathrm{cm} / \mathrm{s})$ & $13.90 \pm 0.83$ & $9.01 \pm 0.84$ & $<0.0001$ \\
\hline (T) E'/A' & $0.91 \pm 0.14$ & $1.78 \pm 0.23$ & $<0.0001$ \\
\hline (T) $\mathrm{S}(\mathrm{cm} / \mathrm{s})$ & $10.82 \pm 0.39$ & $12.73 \pm 0.74$ & 0.001 \\
\hline (LV)MPI & $0.49 \pm 0.02$ & $0.31 \pm 0.01$ & $<0.0001$ \\
\hline (RV)MPI & $0.31 \pm 0.03$ & $0.24 \pm 0.02$ & $<0.001$ \\
\hline EF (\%) & $65.9 \pm 3.9$ & $65.7 \pm 3.9$ & 0.79 \\
\hline ESPAP & $21.9 \pm 1.06$ & $22.4 \pm 1.9$ & 0.54 \\
\hline
\end{tabular}

Abbreviations; cIMT=Carotid Intima-Media Thickness, EATT $=$ epicardial adipose tissue thickness, LVEDD=left ventricular end diastolic volume dimension, LVDPWT $=$ left ventricular posterior wall thickness at end diastole ,IVSDT= interventricular septal thickness at end diastole, LVMI= Left Ventricular Mass Index, (M) $\mathbf{E}=$ trans-mitral peak velocity of early diastolic filling , (M) $\mathbf{A}=$ trans-mitral peak velocity of late diastolic filling , DT=deceleration time of $\mathrm{E}$ wave, (M) $\mathbf{E}^{\prime}=$ mitral annular early diastolic velocity, (M) $\mathbf{A}^{`}=$ mitral annular late diastolic velocity, (M) $\mathbf{S}=$ mitral annular systolic velocity, (T) $\mathbf{E} `=$ tricuspid annular early diastolic velocity, (T) $\mathbf{A}^{`}=$ tricuspid annular late diastolic velocity,(T) $\mathbf{S}=$ tricuspid annular systolic velocity, LVMPI=left ventricular myocardial performance index , RVMPI=right ventricular myocardial performance index , EF $=$ ejection fraction, $\mathbf{E S P A P}=$ Estimated Pulmonary Artery Systolic Pressure .

Having the obese group, there were a statistically significant correlation between the levels of VD and BMI $(\mathrm{r}=-0.97, \mathrm{p}<0.0001), \mathrm{WC}(\mathrm{r}=-0.96, \mathrm{p}<$ $0.0001), \operatorname{SBP}(\mathrm{r}=-0.96, \mathrm{p}<0.0001), \operatorname{DBP}(\mathrm{r}=-$ $0.95, \mathrm{p}<0.0001 \operatorname{EATT}(\mathrm{r}=-0.95, \mathrm{p}<0.0001)$, and cIMT( $r=-0.96, p<0.0001)$. Moreover, VD showed statistically significant correlation with the levels of TG $(\mathrm{r}=0.73, \mathrm{p}<0.000), \mathrm{TC}(\mathrm{r}=-0.61$, $\mathrm{p}<0.000)$, LDL $(\mathrm{r}=-0.59, \mathrm{p}<0.0001), \mathrm{cIMT}(\mathrm{r}=-$ $0.97, \mathrm{p}<0.0001)$, and EATT( $\mathrm{r}=-0.96, \mathrm{p}<0.0001)$ in the non-obese group. Consequently, the levels of EATT showed significant correlation with the level of BMI $(r=0.96, p<0.0001), W C(r=0.97, p<$ $0.0001), \operatorname{SBP}(r=0.96, \mathrm{p}<0.0001), \operatorname{DBP}(\mathrm{r}=0.97$, 
$\mathrm{p}<0.0001)$, fasting insulin( $\mathrm{r}=0.92, \mathrm{p}<0.0001)$, and $\operatorname{cIMT}(\mathrm{r}=0.99, \mathrm{p}<0.0001)$, among the obese group. Furthermore, among non-obese group, EATT as significantly correlated with the levels of TG (r=0.73, p< 0.0001), TC (r=0.62, p< $0.0001)$, adiponectin $(\mathrm{r}=-0.96, \mathrm{p}<0.0001)$, and cIMT $(r=-0.99, p<0.0001)$ (Table 3).

\begin{tabular}{|c|c|c|c|c|c|c|c|c|}
\hline \multirow[t]{3}{*}{ Variables } & \multicolumn{4}{|c|}{$25[\mathrm{OH}] \mathrm{VD}(\mathrm{ng} / \mathrm{ml})$} & \multicolumn{4}{|c|}{ EATT $(\mathbf{m m})$} \\
\hline & \multicolumn{2}{|c|}{ Obese group } & \multicolumn{2}{|c|}{ Non-obese group } & \multicolumn{2}{|c|}{ Obese group } & \multicolumn{2}{|c|}{ Non-obese group } \\
\hline & $\begin{array}{c}\mathbf{r} \\
\text { value }\end{array}$ & $\begin{array}{c}\mathbf{P} \\
\text { value }\end{array}$ & $\begin{array}{c}\mathbf{r} \\
\text { value }\end{array}$ & $\begin{array}{c}\mathbf{P} \\
\text { value }\end{array}$ & $\begin{array}{c}\mathbf{r} \\
\text { value }\end{array}$ & $\begin{array}{c}\mathbf{P} \\
\text { value }\end{array}$ & $\begin{array}{c}\mathbf{r} \\
\text { value }\end{array}$ & $\begin{array}{c}P \\
\text { value }\end{array}$ \\
\hline BMI $\left(\mathrm{Kg} / \mathbf{m}^{2}\right)$ & -0.97 & $<0.0001 *$ & 0.16 & $0.28 * *$ & 0.96 & $<0.0001^{*}$ & 0.21 & $0.18 * *$ \\
\hline WC (Cm) & -0.96 & $<0.0001 *$ & 0.06 & $0.70 * *$ & 0.89 & $<0.0001^{*}$ & -0.09 & $0.58 * *$ \\
\hline SBP (mmHg) & -0.96 & $<0.0001^{*}$ & 0.05 & $0.75 * *$ & 0.97 & $<0.0001^{*}$ & -0.11 & $0.48 * *$ \\
\hline DBP (mmHg) & -0.95 & $<0.0001 *$ & 0.03 & $0.85 * *$ & 0.96 & $<0.0001^{*}$ & -0.07 & $0.63 * *$ \\
\hline FPG (mg/dl) & -0.97 & $<0.0001^{*}$ & 0.09 & $0.55 * *$ & 0.97 & $<0.0001^{*}$ & -0.04 & $0.81 * *$ \\
\hline 2hppbs (mg/dl) & -0.93 & $<0.0001^{*}$ & 0.05 & $0.75 * *$ & 0.93 & $<0.0001^{*}$ & -0.05 & $0.73 * *$ \\
\hline HbA1C\% & -0.87 & $<0.0001 *$ & 0.05 & $0.73 * *$ & 0.87 & $<0.0001^{*}$ & -0.01 & $0.97 * *$ \\
\hline F. insulin $(\mu \mathrm{IU} / \mathrm{ml})$ & -0.92 & $<0.0001 *$ & 0.15 & $0.32 * *$ & 0.92 & $<0.0001^{*}$ & 0.22 & $0.15 * *$ \\
\hline HOMA-IR & -0.95 & $<0.0001^{*}$ & 0.11 & $0.47 * *$ & 0.95 & $<0.0001 *$ & 0.18 & $0.30 * *$ \\
\hline TG (mg/dl) & -0.96 & $<0.0001 *$ & -0.69 & $<0.0001^{*}$ & 0.96 & $<0.0001^{*}$ & 0.73 & $<0.0001 *$ \\
\hline TC (mg/dl) & -0.98 & $<0.0001 *$ & -0.61 & $<0.0001^{*}$ & 0.98 & $<0.0001^{*}$ & 0.62 & $<0.0001 *$ \\
\hline HDL-c (mg/dl) & 0.97 & $<0.0001^{*}$ & 0.88 & $<0.0001^{*}$ & -0.97 & $<0.0001^{*}$ & -0.89 & $<0.0001^{*}$ \\
\hline LDL-c (mg/dl) & -0.98 & $<0.0001^{*}$ & -0.59 & $<0.0001^{*}$ & 0.98 & $<0.0001^{*}$ & 0.70 & $<0.0001 *$ \\
\hline $25[\mathrm{OH}] \mathrm{VD}(\mathrm{ng} / \mathrm{ml})$ & 1 & 1 & 1 & 1 & -0.95 & $<0.0001^{*}$ & -0.96 & $<0.0001^{*}$ \\
\hline iPTH (pg/ml) & -0.98 & $<0.0001 *$ & -0.96 & $<0.0001^{*}$ & 0.98 & $<0.0001^{*}$ & 0.95 & $<0.0001^{*}$ \\
\hline H.S. cRP (mg/dl) & -0.93 & $<0.0001 *$ & -0.27 & $0.08 * *$ & 0.93 & $<0.0001 *$ & 0.27 & $0.08 * *$ \\
\hline Adiponectin $(\mu \mathrm{g} / \mathrm{ml})$ & 0.96 & $<0.0001^{*}$ & 0.95 & $<0.0001 *$ & -0.96 & $<0.0001 *$ & -0.96 & $<0.0001^{*}$ \\
\hline cIMT $(\mathbf{m m})$ & -0.96 & $<0.0001 *$ & -0.97 & $<0.0001^{*}$ & 0.99 & $<0.0001^{*}$ & 0.87 & $<0.0001 *$ \\
\hline EATT (mm) & -0.95 & $<0.0001 *$ & -0.96 & $<0.0001^{*}$ & 1 & 1 & 1 & 1 \\
\hline LVM (g) & -0.91 & $<0.0001^{*}$ & -0.90 & $<0.0001^{*}$ & 0.96 & $<0.0001 *$ & 0.93 & $<0.0001 *$ \\
\hline LVMI $\left(\mathrm{g} / \mathrm{m}^{2.7}\right)$ & -0.73 & $<0.0001 *$ & -0.80 & $<0.0001^{*}$ & 0.80 & $<0.0001^{*}$ & 0.84 & $<0.0001 *$ \\
\hline (M) E/A & 0.97 & $<0.0001^{*}$ & 0.98 & $<0.0001^{*}$ & -0.99 & $<0.0001^{*}$ & -0.95 & $<0.0001^{*}$ \\
\hline DT (m.sec) & -0.96 & $<0.0001^{*}$ & -0.99 & $<0.0001^{*}$ & 0.95 & $<0.0001^{*}$ & 0.97 & $<0.0001 *$ \\
\hline$(\mathbf{M}) \mathbf{E}^{\prime} / \mathbf{A}^{\prime}$ & 0.97 & $<0.0001^{*}$ & 0.94 & $<0.0001 *$ & -0.97 & $<0.0001^{*}$ & -0.94 & $<0.0001 *$ \\
\hline (M) S (cm/s) & 0.97 & $<0.0001 *$ & 0.94 & $<0.0001^{*}$ & -0.98 & $<0.0001 *$ & -0.94 & $<0.0001^{*}$ \\
\hline$(\mathbf{L V}) \mathbf{E} / \mathbf{E}$ & -0.90 & $<0.0001^{*}$ & -0.51 & $0.0004 *$ & 0.89 & $<0.0001^{*}$ & 0.55 & $<0.0001^{*}$ \\
\hline (T) E'/A' & 0.98 & $<0.0001 *$ & 0.98 & $<0.0001 *$ & -0.98 & $<0.0001 *$ & -0.96 & $<0.0001 *$ \\
\hline (T) $\mathrm{S}(\mathrm{cm} / \mathrm{s})$ & 0.98 & $<0.0001^{*}$ & 0.97 & $<0.0001^{*}$ & -0.98 & $<0.0001^{*}$ & -0.91 & $<0.0001^{*}$ \\
\hline (RV) MPI & -0.94 & $<0.0001^{*}$ & -0.77 & $<0.0001 *$ & 0.99 & $<0.0001 *$ & 0.93 & $<0.0001 *$ \\
\hline (LV) MPI & -0.93 & $<0.0001^{*}$ & -0.77 & $<0.0001 *$ & 0.97 & $<0.0001^{*}$ & 0.85 & $<0.0001 *$ \\
\hline EF\% & -0.05 & 0.76 ** & -0.09 & $0.66 * *$ & 0.004 & $0.978 * *$ & 0.21 & $0.18 * *$ \\
\hline ESPAP (mmHg) & -0.10 & $0.53 * *$ & -0.07 & $0.64 * *$ & 0.05 & $0.763 * *$ & -0.04 & $0.81 * *$ \\
\hline \multicolumn{9}{|c|}{ 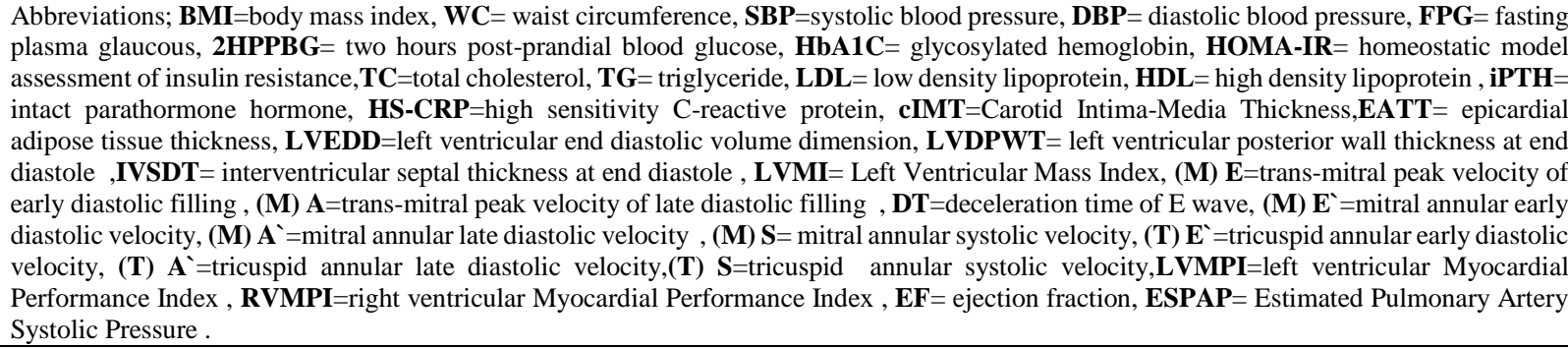 } \\
\hline
\end{tabular}

Multivariate prediction analysis model was done to delineate the independent predictors of EATT in adolescents with MetS subgroup and showed that; only cIMT achieved statistically significant ability in prediction of EATT $(\mathrm{R}=0.75, \mathrm{P}=0.02)$ (Figure.1). 


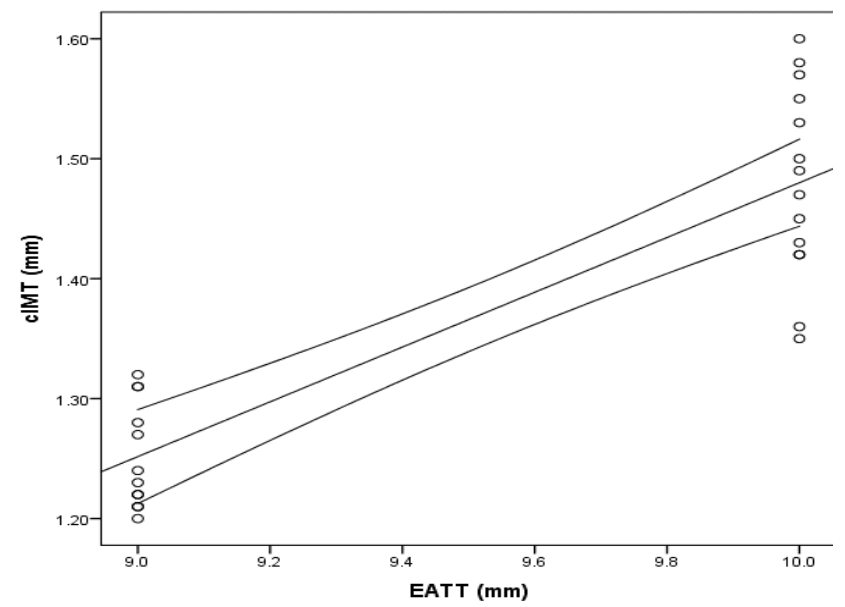

Fig.1: The ability of cIMT in the prediction of EATT among patients with metabolic syndrome.

Having receiver operating characteristics curve (ROC) analysis, of EATT, cIMT, HOMA-IR and s.Adiponectin showed high diagnostic ability in
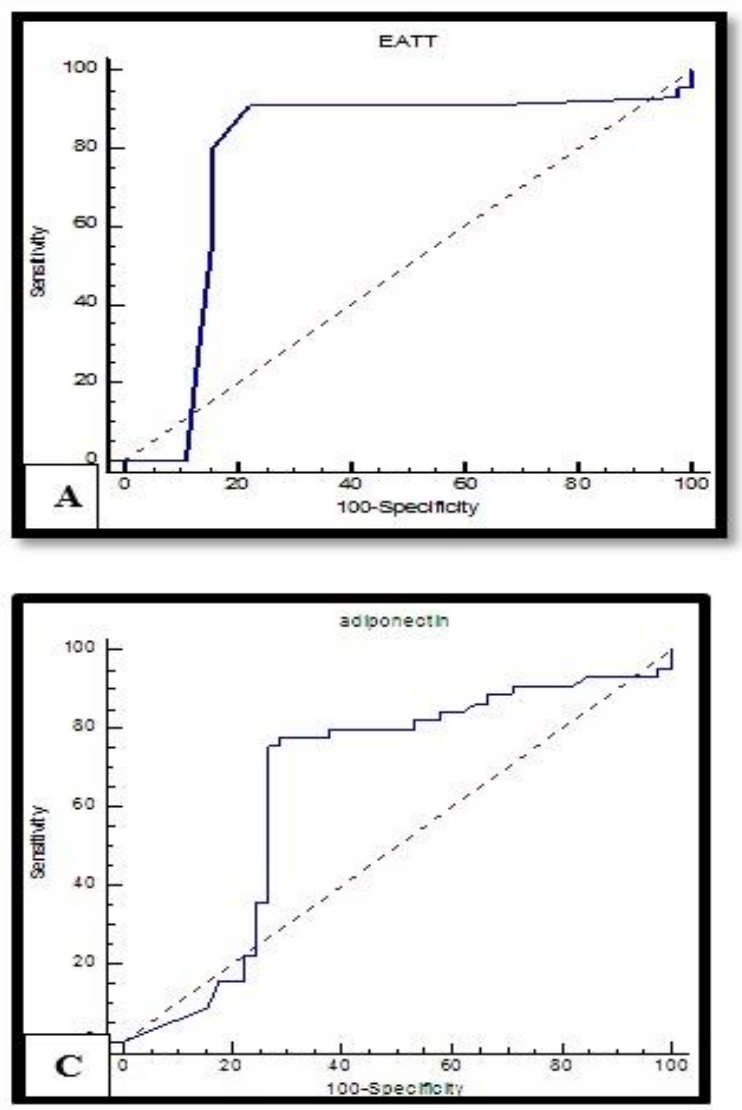

the detection of obesity with specificity of $71.1 \%$, $73.3 \%, 77.8 \%$, and $88.9 \%$, respectively (Figure.2).
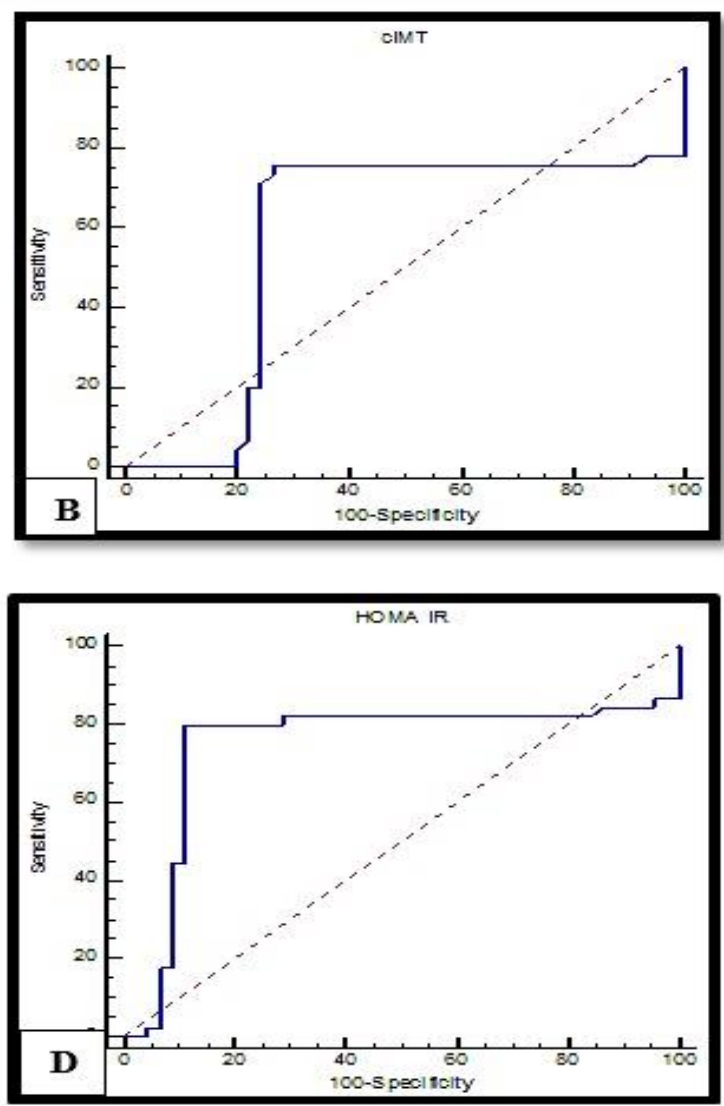

Fig. 2: Receiver operating characteristics (ROC) curves showing the diagnostic ability of (A) EATT, (B) cIMT ,(C) s.Adiponectin (D) HOMA-IR for predicting adolescence obesity.

Furthermore, EATT, cIMT, HOMA-IR and s.Adiponectin showed high diagnostic ability in the detection of Mets with specificity of $77 \%$, $82 \%, 85.4 \%$, and $93.4 \%$, respectively. (Table.4) 
Table 4: Cutoff values, specificity, and sensitivity of EATT, cIMT, HOMA-IR \& s.Adiponectin in prediction of Adolescence obesity and metabolic syndrome.

\begin{tabular}{|c|l|c|c|c|c|}
\hline \multirow{5}{*}{$\begin{array}{c}\text { Adolescence } \\
\text { obesity }\end{array}$} & variable & s.Adiponectin & cIMT & EATT & HOMA-IR \\
\cline { 2 - 5 } & AUC & 0.65 & 0.57 & 0.78 & 0.74 \\
\cline { 2 - 6 } & Cut-off value & $13.49(\mu \mathrm{g} / \mathrm{ml})$ & $0.76(\mathrm{~mm})$ & $6(\mathrm{~mm})$ & 3.4 \\
\cline { 2 - 6 } & Sensitivity (\%) & 77.7 & 75.6 & 91.1 & 80 \\
\cline { 2 - 6 } & Specificity (\%) & 71.1 & 73.3 & 77.8 & 88.9 \\
\cline { 2 - 6 } & (+ve) Likelihood ratio & 0.31 & 2.83 & 4.10 & 7.20 \\
\cline { 2 - 6 } & (-ve) Likelihood ratio & 2.69 & 0.33 & 0.1 & 0.2 \\
\cline { 2 - 6 } & PPV (\%) & 23 & 23.9 & 31.3 & 44.4 \\
\cline { 2 - 6 } & NPV (\%) & 96.6 & 96.4 & 98.7 & 97.6 \\
\hline \multirow{4}{*}{$\begin{array}{c}\text { Adolescence } \\
\text { syndabolic }\end{array}$} & AUC & 0.7 & 0.54 & 0.77 & 0.67 \\
\cline { 2 - 6 } & Cut-off value & $10.09(\mu \mathrm{g} / \mathrm{ml})$ & $1.05(\mathrm{~mm})$ & $8(\mathrm{~mm})$ & 5.1 \\
\cline { 2 - 6 } & Sensitivity (\%) & 86.2 & 69 & 88.46 & 69 \\
\cline { 2 - 6 } & Specificity (\%) & 77 & 82 & 85.4 & 93.4 \\
\cline { 2 - 6 } & (+ve) Likelihood ratio & 3.76 & 3.82 & 18.23 & 10.5 \\
\cline { 2 - 6 } & (-ve) Likelihood ratio & 0.18 & 0.38 & 0.1 & 0.33 \\
\cline { 2 - 6 } & PPV (\%) & 29.4 & 29.8 & 66.9 & 96.4 \\
\cline { 2 - 6 } & NPV (\%) & 98 & 96 & 98.8 & 53.9 \\
\hline
\end{tabular}

Abbreviations; EATT $=$ epicardial adipose tissue thickness, $\mathbf{c I M T}=$ Carotid Intima-Media Thickness, HOMA-IR= homeostatic model assessment of insulin resistance $\mathbf{A U C}=$ area under curve, $\mathbf{P P V}=$ positive predictive value, $\mathbf{N P V}=$ negative predictive value,

\section{Discussion}

Obesity is a multifaceted health problem with multisystem consequences and its spectrum not restricted to adulthood. Occurrence of obesity during adolescence and its persistence during the adulthood usually exists and is contributing significantly in increasing the risks of various morbidities and mortalities related to cardiac dysfunction $^{(16)}$.

The evidence retrieved in our study illustrated that; obese adolescents were different substantially from non-obese adolescents regarding their anthropometric measurements, laboratory, and echocardiographic structural and functional parameters with a high prevalence of cardiometabolic risk factors including; elevated $\mathrm{BP}$, impaired fasting glucose \& glucose tolerance, insulin resistance. Moreover, the prevalence of metabolic syndrome was $(57.8 \%)$. They exhibited also a subtle, sub-diagnostic, impairment of both systolic and diastolic functions (Tables 1\&2).

This confirms our postulation that early onset obesity tends to affect the cardiovascular system adversely by creating a set of different pathological processes including premature and accelerated wide-spread thermogenesis, low grade generalized inflammatory reactions that impair the endothelial functions, impaired metabolic pathways leading to insulin resistance and dyslipidemia, alteration in the humoral factors released from the adipose tissue, the adipokines, with increased synthesis and release of the harmful adipokines as well as the proinflammatory cytokines in association with down regulation of the protective ones. All of the aforementioned mechanisms culminated the impairment of cardiovascular functions as well as the adverse geometrical remodeling detected among obese adolescents included in our study. Despite BMI is feasible procedure in the assessment of patients at high risk of cardiometabolic dysfunction, it does not discriminate the distribution of body fat as it reflects only fat and lean body masses $^{(17)}$.Thereafter, assessment of visceral adiposity as the abdominal adiposity using EATT is more crucial than general adiposity ${ }^{(\mathbf{1 8})}$.

For that, we chose the EAT as an interesting depot of visceral adipose tissue with close proximity to the heart to study its impact on the myocardial structure and functions trying to reproduce the utility of measuring its thickness by transthoracic echocardiography as an easily 
obtainable, non-invasive and reproducible method for evaluating the underlying cardiometabolic state in obese adolescents trying to illustrate the role of visceral adiposopathy in cardiometabolic dysfunction.

Our results pointed to the usefulness of EATT measurement as an indicator of cardiovascular structure and function supported by many statistical evidences; firstly, EATT was found to be significantly higher in obese adolescents compared to the non-obese ones. Secondly, EATT showed a statistically significant positive correlations with all the cardiometabolic risk factors including obesity, elevated BP, impaired fasting glucose \& glucose tolerance, insulin resistance, cIMT which is a marker of subclinical atherosclerosis, the deterioration of both systolic and diastolic functions (Table 3). Thirdly, EATT showed high diagnostic ability in the detection of adolescence obesity and MetS at cut off values $(6 \mathrm{~mm}) \&(8 \mathrm{~mm})$ respectively with sensitivity of $91.1 \%$ and specificity of $77.8 \%$ in case of obesity while with sensitivity of $88.46 \%$ and specificity of $85.4 \%$ in case of adolescence MetS (Table 4). Our results were concomitant with previous studies. For instance, Elshorbagy et al, notified that; EATT showed significant correlation with the anthropometric parameters such as BMI, and WC and laboratory variables such as fasting insulin, TG, and Hs-CRP among obese group and specially in patients with MetS ${ }^{(\mathbf{1 9})}$. Moreover, this results also in conformity with Akyol et al. that illustrated the presence of cardiometabolic dysfunction with increased EATT, cIMT , LVMI, and MPI were found in adolescents obese patients (20).

Our study have found in the multivariate prediction analysis model that was done to delineate the independent predictors of EATT in adolescents with MetS subgroup that; only cIMT achieved statistically significant ability in prediction of EATT $(\mathrm{R}=0.75, \mathrm{P}=0.02)$.

The significant correlation between EATT and cIMT brings to light that; EATT is a promising biomarker in assessment of cardiac dysfunction relative to $\mathrm{WC}$, and BMI among adolescent's obese patients owed by its close relation to the severity of carotid artery stiffness, and endothelial dysfunction ${ }^{(21)}$.However, this result should by subjected to further evaluation.
VDD was associated with obesity and related diseases that hinder patient's quality life ${ }^{(22)}$. In our work, the levels of VD showed significant negative correlations with all the cardiometabolic risk factors including obesity, elevated BP, impaired fasting glucose \& glucose tolerance, presence of MetS, insulin resistance, cIMT, the deterioration of both systolic and diastolic functions among the adolescents obese group.

Our result was in conformity with previous studies. Pacifico et al. illustrated the significant association of VD levels and obesity, elevated blood pressure, and $\mathrm{MetS}^{(\mathbf{2 3})}$. The underlying mechanism is a doubtful issue despite the numerous studies that showed the inverse association between VD levels and body fat mass, elevated BP, elevated glaucous, and TG level ${ }^{(24}$, ${ }^{25)}$.However, the inverse correlation between VD levels and insulin resistance may provide a possible explanation ${ }^{(26)}$. Furthermore, it was established that obesity is associated with insulin resistance, low HDL, TC, and high LDL. However, VDD may be a sequel of obesity itself $^{(27)}$. Throughout the past era, it was suggested that VD has a protective role against cardiovascular dysfunction, (28) our results were agreed with the previous evidence particularly with the close association of VD levels and the echocardiological parameters. This result may be explained due to the reno-protective function (suppression of renin angiotensin system), and its roles in calcium metabolism, and on the vascular wall $^{(28)}$.

To the best of our knowledge, no previous study had investigated the possible association between EATT and the level of 25[OH]VD particularly in adolescence. Under this condition, we demonstrated for the first time an evidence of the inverse relationship between those 2 parameters in the obese adolescent population.

In contrast to our findings, Utku et al. studied the relationship between EATT and VD in patients with metabolic syndrome and found that EATT increased in patients with metabolic syndrome. In contradiction to literature; the levels of $25[\mathrm{OH}] \mathrm{VD}$ was not found to be low in patients with metabolic syndrome. Any significant correlation was not found between EATT and $25[\mathrm{OH}] \mathrm{VD}$ levels. However, this study was conducted on adults with their mean of ages is $38.5 \pm 9.7^{(29) \text {. }}$ 


\section{Limitations of our study}

The main limitation of the present study is the smaller sample size that prevented the conduction of further subgroup analysis particularly we demonstrated the presence of the different obesity phenotypes among the obese group. Further large-scale studies with larger sample size are warranted to solve this issue. Another limitation encountered is that we were not taking the seasonal variations during sampling for $25[\mathrm{OH}] \mathrm{VD}$ levels in consideration which is a known factor that can affect the 25[OH]VD level assay.

\section{Conclusions}

It is of interest and is not redundant to use EATT as a marker for the adverse metabolic state and cardiovascular dysfunction among obese adolescents; VD deficiency is closely related to the classical cardiometabolic risk factors including obesity, elevated blood pressure, diabetes mellitus and prediabetes, dyslipidemia during the adolescence. Therefore, obese adolescents should be subjected to close monitoring and high levels of health care to avoid short term, and long-term cardiometabolic dysfunctions.

\section{References}

1.Collaborators GO (2017): Health effects of overweight and obesity in 195 countries over 25 years. New England Journal of Medicine, 377(1):13-27.

2.I'allemand D, Wiegand S, Reinehr T et al. (2008): Cardiovascular risk in 26,008 European overweight children as established by a multicenter database. Obesity,16(7): 1672-9.

3.Rankin J, Matthews L, Cobley S et al. (2016): Psychological consequences of childhood obesity: psychiatric comorbidity and prevention. Adolescent health, medicine and therapeutics, 7(1): 125 .

4.Twig G, Yaniv G, Levine $\mathrm{H}$ et al. (2016): Body-mass index in 2.3 million adolescents and cardiovascular death in adulthood. New England Journal of Medicine,374(25):2430-40.

5.Carolan-Olah M, Duarte-Gardea M, Lechuga J (2015): A critical review: early life nutrition and prenatal programming for adult disease. Journal of clinical nursing, 24(23-24) :3716-29.

6.Reaven GM (1991): Insulin resistance and compensatory hyperinsulinemia; role in hypertension, dyslipidemia, and coronary heart disease. American heart journal, 121(4) :1283-8. 7.Zimmet P, Alberti KGM, Kaufman F et al. (2007): The metabolic syndrome in children and adolescents-an IDF consensus report. Pediatric diabetes, 8(5) :299-306.

8.Eller NH and Netterstrom B (2001): The intima media thickness and coronary risk factors. International angiology, 20(2):118.

9.Gastaldelli A and Basta G (2010): Ectopic fat and cardiovascular disease: what is the link? Nutrition, Metabolism and cardiovascular diseases, 20(7):481-90.

10.Iacobellis $G$ and Barbaro $G$ (2008): The double role of epicardial adipose tissue as proand anti-inflammatory organ. Hormone and metabolic research, 40(07): 442-5.

11.Ramagopalan SV, Heger A, Berlanga AJ et al. (2010): A ChIP-seq defined genome-wide map of vitamin D receptor binding: associations with disease and evolution. Genome research, 71(1): 398-3.

12.Tanner JM and Whitehouse RH (1976): Clinical longitudinal standards for height, weight, height velocity, weight velocity, and stages of puberty. Archives of disease in childhood,51(3):170-9.

13.Iacobellis $G$ and Willens HJ (2009): Echocardiographic epicardial fat: a review of research and clinical applications. Journal of the American Society of Echocardiography,22(12):1311-9.

14.Schiller NB, Shah PM, Crawford M et al. (1989): Recommendations for quantitation of the left ventricle by two-dimensional echocardiography. Journal of the American Society of Echocardiography, 5: 358-67.

15.Lang RM, Bierig M, Devereux RB et al. (2005): Developed in conjunction with the European Association of Echocardiography, a branch of the European Society of Cardiology. Journal of the American Society of Echocardiography,18(12):1440-63.

16. Cote AT, Harris KC, Panagiotopoulos C et al. (2013): Childhood obesity and cardiovascular dysfunction. Journal of the American College of Cardiology, 62(15): 1309-19.

17.Mason C, Craig CL and Katzmarzyk PT (2008): Influence of central and extremity circumferences on all-cause mortality in men and women. Obesity, 16(12): 2690-5. 
18.Janssen I, Katzmarzyk PT and Ross $R$ (2004): Waist circumference and not body mass index explains obesity-related health risk. The American journal of clinical nutrition,79(3):37984.

19.Elshorbagy HH, Fouda ER, Kamal NM et al. (2016): Evaluation of epicardial fat and carotid intima-media thickness in obese children. Iranian journal of pediatrics. 26(1): 489-18.

20.Akyol B, Boyraz $M$ and Aysoy C (2013): Relationship of epicardial adipose tissue thickness with early indicators of atherosclerosis and cardiac functional changes in obese adolescents with metabolic syndrome. Journal of clinical research in pediatric endocrinology, 5(3): 156.

21.Eroglu S, Sade LE, Yildirir A et al. (2009): Epicardial adipose tissue thickness by echocardiography is a marker for the presence and severity of coronary artery disease. Nutrition, Metabolism and Cardiovascular Diseases, 19(3):211-7.

22.Reis JP, von Mühlen D, Miller ER et al. (2009): Vitamin D status and cardiometabolic risk factors in the United States adolescent population. Pediatrics, 124(3): e371-e9.

23.Pacifico L, Anania C, Osborn JF et al. (2011): Low $25(\mathrm{OH})$ D3 levels are associated with total adiposity, metabolic syndrome, and hypertension in Caucasian children and adolescents. European Journal of Endocrinology,11(1):545.

24.Arunabh S, Pollack S, Yeh J et al. (2003): Body fat content and 25-hydroxyvitamin D levels in healthy women. The Journal of Clinical Endocrinology \& Metabolism,88(1):157-61.

25.Scragg R, Sowers $M$ and Bell $C$ (2007): Serum 25-hydroxyvitamin D, ethnicity, and blood pressure in the Third National Health and Nutrition Examination Survey. American journal of hypertension, 20(7): 713-9.

26.Ford ES, Ajani UA, McGuire LC et al. (2005): Concentrations of serum vitamin $D$ and the metabolic syndrome among US adults. Diabetes care,28(5):1228-30.

27.Osei K (2010): $25-\mathrm{OH}$ vitamin D: is it the universal panacea for metabolic syndrome and type 2 diabetes? Oxford University Press, 19(1):781-12.

28. Vaidya A and Forman John $P$ (2010): Vitamin D and Hypertension. Hypertension, 56(5): 774-9.

29. Utku IK, Okuturlar Y, Demir E et al. (2015): Relationship between epicardial adipose tissue thickness and vitamin D in patients with metabolic syndrome. International journal of clinical and experimental medicine, 8(4):5707. 